\title{
Porous Biomaterials: Classification, Fabrication and Its Applications in Advanced Medical Science
}

\author{
Mosammat Jesmin Sultana ${ }^{1, ~ *}$, Fazle Rabbi Shakil Ahmed ${ }^{2}$ \\ ${ }^{1}$ Department of Materials Science and Engineering, Rajshahi University, Rajshahi, Bangladesh \\ ${ }^{2}$ Department of Pharmacy, Khwaja Yunus Ali University, Sirajgonj, Bangladesh
}

Email address:

jesmin_mse@ru.ac.bd(M. J. Sultana),jssumi8@gmail.com (M. J. Sultana), frshakil@gmail.com (F. R. S. Ahmed)

${ }^{*}$ Corresponding author

\section{To cite this article:}

Mosammat Jesmin Sultana, Fazle Rabbi Shakil Ahmed. Porous Biomaterials: Classification, Fabrication and Its Applications in Advanced Medical Science. American Journal of Nanosciences. Vol. 4, No. 2, 2018, pp. 16-20. doi: 10.11648/j.ajn.20180402.11

Received: September 4, 2018; Accepted: October 4, 2018; Published: October 23, 2018

\begin{abstract}
Biomaterials are natural or synthetic, alive or lifeless, and usually made of multiple components that interact with biological systems. In this article, it has been reported the applications of porous biomaterials in living bodies and its biocompatibility. Biocompatibility is related to the behaviour of biomaterials in various environments under various chemical and physical conditions. The term may refer to specific properties of a material without specifying where or how the material is to be used. Here, declared different types of biomaterials and their fabrication methods. And also studied various applications of porous biomaterials in medical and biological sciences. Now a day, biomaterials are used to make devices to replace a part or a function of the body with the help of advance surgical technique and instruments in safe, reliably economically and physiologically acceptable manner. This study also reported that the biomaterials comprise a varieties of properties such as biocompatible, bioactive, surface reactive, biodegradable, sterilizability, adequate mechanical and physical properties, manufacture ability, low weight and reasonable cost etc. The main focuses of this review was the applications of biomaterials in different parts of body. Replacement parts were heart, lung, eye, ear, bone, kidney, bladder, nerve stimulator and dental applications. Therefore, this article is focusing on three specific topics such as classification, fabrication methods and suitable implant applications in advances medical science.
\end{abstract}

Keywords: Porous Biomaterials, Classification, Fabrication, Porosity, Application, Implant, Medical Sciences

\section{Introduction}

This review article was discussed the function of porous biomaterials in advance medical sciences. Unfortunately, millions of patients are suffering caries, gnashing and osteoporosis lead to deterioration of bone and teeth. There are huge costs for the societies and a great demand for orthopaedic and dental biomaterials implant. Implants have been extensively used in oral rehabilitation and orthopaedics for replacements of partially damaged skeletal tissues [1]. Ideal biomaterials need to simultaneously satisfy many requirements for the applications such as biocompatibility, strength, fatigue, durability, non-toxicity, corrosion resistance, and sometimes aesthetics [2]. The wear characteristics and visco-elastic properties of implants are also important. In the case of bone, materials should preferably be both osteoinductive (capable of promoting the differentiation of progenitor cells down an osteoblastic lineage), osteoconductive (support bone growth and encourage the ingrowth of surrounding bone) and capable of osseointegration (integrate into surrounding bone) [3] Porosity is the percentage of null and void space in a solid and it is a morphological property. Porous biomaterials are the type of biomaterials which has this property [4]. For all porous biomaterials, the important structural parameters is

$$
\text { Total porosity }=\frac{\text { Total pore volume }}{\text { Over all volume }}
$$

Total porosity of the porous biomaterials has negative effect on its mechanical properties. However, total porosity alone does not have a direct connection with tissue in growth, as pore size and pore interconnectivity is more important for 
tissue in growth. Each porous material might have three types of pores such as closed, through and blind pores. The closed pores are not accessible to fluids. The blind pores terminate inside the material. The through pores are those that make possible the complete passageway of fluids. The open porosity includes only through and blind pores. Porosity that includes closed pores has a great impudence on mechanical properties of a material, open porosity has its direct impact in the possibility of penetration desired and undesired fluids, cells or bacteria. Porous metals with an interconnected pore structure are of particular interest for orthopaedic implant applications due to their potential ability to facilitate tissue ingrowth. Pores are necessary for tissue formation, because they allow migration and proliferation of cells, as well as vascularization. In addition, a porous surface improves mechanical interlocking between the implant biomaterial and the surrounding natural tissue, providing greater mechanical stability at this critical interface.

\section{Classification of Porous Biomaterials}

\subsection{Metallic Porous Biomaterials}

"Vanadium steel" was the first developed metal alloy specifically for human which was used to produce bone fracture dishes and screws. The majority of the metals such as iron $(\mathrm{Fe})$, nickel $(\mathrm{Ni})$, cobalt $(\mathrm{Co})$, molybdenum $(\mathrm{Mo})$, chromium $(\mathrm{Cr})$, titanium $(\mathrm{Ti})$, niobium $(\mathrm{Nb})$, tantalum $(\mathrm{Ta})$, and tungsten(W) that were used to compose alloys for manufacturing implants can only be tolerate by the body in little amounts. Sometimes those metallic elements such as iron, cobalt etc are naturally form taking place. Iron is necessary in red blood cell functions and cobalt is necessary for synthesis of a vitamin B-12. But cannot be tolerate in huge amounts in the body [5].

\subsection{Ceramic Porous Biomaterials}

Ceramics are usually hard in fact, the amount of hardness is calibrated against ceramic materials. It is paying attention on a common impression such as bio-inert, bioactive, surface reactive ceramics, biodegradable, restorable and bioceramics. Ceramics are use in fabricating implants which can be classified as non absorbable, bioactive or surface reactive [6] and biodegradable [7]. The most significant properties of ceramic porous biomaterials are non toxic, non inflammatory, non allergic, biocompatible, bio functional and non carcinogenic etc for its life span in the host. Silicone, Nitrides, Alumina, Carbons and Zirconium are the inert ceramic porous biomaterials. Certain glass ceramics are bio reactive, calcium phosphates and calcium aluminates are resorbable ceramics [8].

\subsection{Polymeric Porous Biomaterials}

Synthetic polymeric porous biomaterials have been commonly used in prosthetic materials, dental materials, medical disposable supplies, extra corporeal devices, implants, dressings, encapsulates, polymeric drug delivery systems, tissue engineered products and ortho doses like those of metal and ceramics substituents. The main advantages of the polymeric porous biomaterials compared to metal or ceramic materials are firstly manufacturability to produce various shapes (latex, film, sheet, fibers etc.), secondly process ability, reasonable cost and availability with desired mechanical and physical properties. The required properties of polymeric porous biomaterials are similar to other biomaterials. There are sterilizability, biocompatibility, adequate mechanical, physical properties and manufacture ability [9].

\subsection{Composite Porous Biomaterials}

Bone, wood, dentin, cartilage and skin are the natural composites. Natural foams include lung, cancellous bone and wood are also natural composites. Natural composites often exhibit hierarchical structures in which porous, particulate and fibrous structural features are seen on different microscales [10]. Applications of porous composites in biomaterials are dental filling composites, bone cement and orthopaedic implants.

\section{Materials and Methods}

The applications of porous biomaterials depend on its porosity, pore size and other porous parameters. But the fabrication of porous materials with different pore sizes depend different manufacturing processes. Several methods have been developed for the fabrication of porous biomaterials. They are summarized below

\subsection{Sintering of Graded Particle}

In this method biomaterials are good pore interconnectivity but small pore size and low porosity [11].

\subsection{Vacuum in Filtration}

In this process biomaterials are good pore interconnectivity and control pore size but it has dimensional limitation [12].

\subsection{Pressure Filtration of Mixed Particles}

Pressure filtration of mixed particles has convenient porosity but poor pore interconnectivity and complicated process $[13,14]$.

\subsection{Infiltration of Compression Molded Sponge}

Infiltration of compression moulded sponge has high pore interconnectivity, graded pore size, porosity and pore shape $[15,16]$.

\subsection{Multiple Tape Casting \& Lamination}

In this process biomaterials are controllable pore size and porosity but poor interconnectivity especially on the interfaces of pore [17].

There are also various processing techniques such as partial sintering, sacrificial fugitives, foaming, freeze casting, metal injection moulding and rapid prototyping etc [18]. 


\section{Applications of Porous Biomaterials in Advanced Medical Sciences}

The use of biomaterials in different parts of body and replacement of different parts for living being are described below:

\subsection{Heart}

\subsubsection{Artificial Pacemaker}

A pacemaker is a therapeutic apparatus that uses electrical impulses, deliver by electrodes contracting the heart strength to normalize the beating of the heart. The most important purpose of a pacemaker is to maintain a satisfactory heart rate, either because the heart's natural pacemaker is not fast enough or there is a block in the heart's electrical conduction system. Others have multiple electrodes stimulating differing positions within the heart to improve synchronization of the lower chambers (ventricles) of the heart [19].

\subsubsection{Artificial Heart Valve}

An artificial heart valve is a device implanted in the heart of a patient with the disease of valvular heart. Porous biomaterials have been commonly used to manufacture these devices. Natural heart valves become dysfunctional for a variety of pathological causes. Some pathologies may require complete surgical alternative of the natural heart valve with a heart valve prosthesis [20].

\subsubsection{Artificial Heart}

An artificial heart is a medical device which is use for the replaces of the heart. Artificial hearts are naturally used to bridge the time to heart transplantation or to permanently replace the heart in case heart transplantation is not possible. Although other similar inventions preceded it going back to the late 1940s, the first artificial heart to be successfully implanted in a human was the Jarvik-7 designed by a team including Robert Jarvikand implemented in 1982 [21].

\subsection{Lung}

\section{Oxygenator machine}

An oxygenator is a medical device which is capable of exchange oxygen and carbon dioxide in the blood of the patient during surgical actions that may require the interruption or termination of blood flow a critical organ or great blood vessel in the body [22]. Porous biomaterials are commonly used in oxygenator machine

\subsection{Eye}

\subsubsection{Contact Lens}

A contact lens which manufacture by porous biomaterials. It is a thin lens placed directly on the surface of the eye. Contact lenses are considered as medical devices and can be damaged to correct vision or for cosmetic or therapeutic reasons.

\subsubsection{Intraocular Lens}

An intraocular lens is a lens implanted in the eye which is use to the disease of cataracts or myopia. The most common type of intraocular lens is the pseudophakicintraocular lens. The pseudophakicintraocular lens is replaces the original crystalline lens and provide the light focusing function originally under taken by the crystalline lens. Intraocular lens usually consist of a small plastic lens with plastic side strut, called haptics which hold the lens in place within the capsular bag inside the eye [23].

\subsection{Ear}

\section{Cochlear implant}

A artificial cochlear implant (CI) which manufactured by porous biomaterials is a surgically implanted electronic device that provides a sense of sound to a person who is profoundly deaf or severely hard of hearing. Cochlear implants may help to provide hearing in patients who are unable to hear because of injure to sensory hair cells in their cochleas. In those patients, the implants often can enable sufficient hearing for better understanding of speech.

\subsection{Bone}

\subsubsection{Artificial Bone Plate}

Iron and steel were the most widely in use materials because of their tensile strength which made iron and steel attractive. They dissolved rapidly and aggravated erosion of adjacent bone. From the point of view of erosion, biocompatibility and fatigue life, stainless steel is inferior to other super alloys. However, it still may have applications in old patients, in whom physical demands and life expectancy are limited. And especially when cost is a major determinant. Stainless steels are suitable to use only in temporary implant devices.

\subsubsection{Intra Medullary Rod}

An intramedullary rod is a medical device which is constructing by biomaterials. Intramedullary rod also known as an intra-medullary nail or inter-locking nail is a metal rod forced into the medullary cavity of a bone. Intramedullary nails have extended been used to treat fractures of bones of the body [24].

\subsection{Kidney}

\section{Catheters}

A catheter is a thin tube extruded as medical grade materials. In medicine, it is serving a broad range of functions. Catheters are medical devices that can be inserted in the body to treat diseases or perform a surgical procedure. By modifying the material or adjusting the technique catheters are manufactured. It is possible to tailor catheters for gastrointestinal, cardiovascular, neurovascular, urological and ophthalmic applications.

\subsection{Bladder}

\subsubsection{Stent}

In the technical vocabulary of medicine, a stent is a mesh tube inserted into a natural passage in the body to prevent or counteract a disease. The term may also refer to a tube used 
to temporarily hold such a natural conduit open to allow access for surgery. The most common use of stent is in the coronary arteries such as a drug eluting stent, a bioabsorbable stent, a dual-therapy stent (combination of both drug and bioengineered stent) and occasionally a covered stent is also insert [25].

\subsubsection{Nerve Stimulator}

Nerve stimulator is an electronic device that stimulates the nerve by passing through the electric current into the body and in this procedure current is used to treat chronic pain. There are two related forms of electrical stimulation generally used to treat chronic pain. Spinal cord stimulation and peripheral nerve stimulation are these two types of electrical nerve stimulation. Moreover, a small pulse maker sends electrical pulses to the peripheral nerve stimulation or to the spinal cord stimulation. These pulses interfere with the nerve impulses that create pain. Those nerve stimulators are also constructed by biomaterials.

\subsection{Dental Applications}

Porous materials can be used to establish an effective means of implant stabilization by tissue in growth. It has been shown that severe caution should be exercised in applying this concept to dental implants placed per imucosally. The micro-porosity of the surface is results in an inflammatory reaction that prevents formation of an effective biological seal. Observations show that an effective biological seal can not be established with materials possessing crown and cervical surface micro-porosity.

\section{Conclusion}

There are many examples of actual applications of the porous biomaterials implant. The interest of searching a new processing methods and new field of application of the materials still continues. So far, many fabrication methods have concept of porous biomaterials has led to the development of implant been developed for producing porous biomaterials and in many body systems where these are used and made our life longer and comfortable to live. Over the years, a variety of applications have been developed, resulting in porous biomaterials implant that can address unresolved clinical problems. And in future will do so. This paper intends to systematically review the processing techniques used in porous biomaterial implant and also including ceramics, polymers, metals, and glasses along with their mechanical performances.

\section{Acknowledgements}

Jesmin Sultana is grateful to the Department of Materials Science \& Engineering in Rajshahi University, Bangladesh for the technical support and other facilities of this review article. Fazle Rabbi Shakil Ahmed was helped in this article by his technical support.

\section{References}

[1] Barrère, F., Mahmood, T. A., de Groot K., van Blitterswijk C. A., Advanced biomaterials for skeletal tissueregeneration: Instructive and smart functions. Mater. Sci. Eng. R: Reports. 59(2008)538-71.

[2] Yang, J., Xiang, H. J., A three-dimensional finite element study on the biomechanical behavior of an FGBM dental implant in surrounding bone, J. Biomech. 40(2007)2377-2385.

[3] Stevens, M. M. Biomaterials for bone tissue engineering. Mater. Today 11(2008)18-25.

[4] Meenakshi, Mour, Debarun, Das, Thomas, Winkler, Elisa, Hoenig Gabriela, Mielke Michael, M, Morlock, Arndt, F, Schilling, Advances in Porous Biomaterials for Dental and Orthopedic Applications, Materials. 3(2010)2947-2974.

[5] A. Srivastav, An Overview of Metallic Biomaterials for Bone Support and Replacement, Biomedical Engineering, Trends in Materials Science, 153-168,

[6] W, C, Billotte, Ceramic Biomaterials,(2006)339:1-39.

[7] Tetsuya Tateish, Biomaterials in Asia: In Commemoration of the 1st Asian Biomaterials, ISBN: 13-978-981-283-574-1.

[8] Anthony Atala, Principles of Regenerative Medicine, ISBN: 978-0-12-369410-2.

[9] Hai, Bang, Lee, Gilson, Khang, Jin, Ho, Lee, Polymeric Biomaterials, book: Biomedical Engineering Fundamentals. 40(24) (2006)1-40.

[10] Hermann Ehrlich, Biological Materials of Marine Origin, (2010) ISBN: 978-90-481-9129-1.

[11] Thieme, M., Wieters, K. P., Bergner, F., Scharnweber, D., Worch, H., Ndop, J., Kim, T. J., Grill, W., Titanium powder sintering for preparation of a porous functionally graded material destined for orthopaedic implants, J. Mater. Sci. 12 (2001)225-231.

[12] Miao X., Hu Y., Liu J., Tio B, Cheang P., Khor K. A., Highly interconnected and functionally graded porous Bioceramics, Key Eng. Mater. (2003)595-598.

[13] Iwata, M., Shimono, A., Kishiro, K., Kunieda Y., Preparation of porous hydroxyapatite materials with a continuous porosity profile by use of a filtration method, J. Jpn. Inst. Metal. 62(1998)1088-1094.

[14] Droschel, M., Hoffmann, M. J., Oberacker, R., Both, H. V., Schaller, W., Yang, Y. Y.; Munz, D. SiC-ceramicswith tailored porosity gradients for combustion chambers, Key Eng. Mater 175-176(2000)149-162.

[15] Corbin, S. F.; Zhao, X., Henein, H., Apte P. S., Functionally graded metal/ceramic composites by tape casting, lamination and infiltration., Mater. Sci. Eng. A. 262(1999)192-203.

[16] Cichocki, F. R., Jr., Trumble, K. P., Rodel, J. Tailored porosity gradients via colloidal infiltration of compression-molded sponges, J. Amer. Ceram. Soc. 81(1998)1661-1664.

[17] Werner, J. P., Linner-Krcmar, B., Friess, W., Greil, P., Mechanical properties and in vitro cell compatibility of hydroxyapatite ceramics with graded pore structure, Biomaterials. 23(2002)4285-4294 
[18] Elham Babaie and Sarit B. Bhaduri. Fabrication Aspects of Porous Biomaterials in Orthopedic Applications: A Review, ACS Biomater. Sci. Eng. 4(1)(2018)1-39.

[19] McWilliam JA, (1899). "Electrical stimulation of the heart in man". BrMedJ 1 (1468):348. Doi:10.1136/bmj.1.1468.348. "Electrical Stimulation of the Heart in Man - 1899", Accessed Jan 27, (2014).

[20] Pibarot, P., Dumesnil, J. G., Prosthetic Heart Valves: Selection of the Optimal Prosthesis and Long-Term Managemen, Circulation. 119(2009)1034-48.

[21] J. Wei, K. K. Cheng, D. Y. Tung, C. Y. Chang, W. M. Wan, Y. C. Chuang: Successful Use of Phoenix-7 Total Artificial Heart. Transplantation Proceedings. 30(1998)3403-4.
[22] Cardiotomy Suction: A Major Source of Brain Lipid Emboli During Cardiopulmonary Bypass" Ann Thorac Surg. 65(1998)1651-1655.

[23] Sanders, Donald, Vukich, John A. Comparison of Implantable Collamer Lens (ICL) and Laser-assistedin Situ Keratomileusis (LASIK) for Low Myopia, Cornea. 25 (10) (2006)1139-46.

[24] Lefaivre, K. A.; Guy, P.; Chan, H.; Blachut, P. A. Long-Term Follow-up of Tibial Shaft Fractures Treated with Intramedullary Nailing. Journal of Orthopaedic Trauma 22 (8) (2008)525-529.

[25] Vogel, T, Shindelman, L., Nackman, G., Graham, A. Efficacious Use of Nitinol Stentsinthe Femoraland Popliteal Arteries, Journal of Vascular Surgery. 38(6)(2003)1178-1183. 